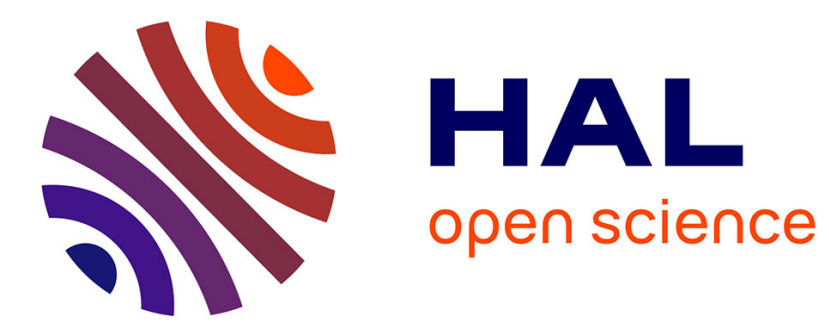

\title{
Motorcycle Maximal Safe Speed in Cornering Situation
}

Hamid Slimi, Dalil Ichalal, Hichem Arioui, Saïd Mammar

\section{To cite this version:}

Hamid Slimi, Dalil Ichalal, Hichem Arioui, Saïd Mammar. Motorcycle Maximal Safe Speed in Cornering Situation. 10th IEEE International Conference on Networking, Sensing and Control (ICNSC 2013), Apr 2013, Evry, France. pp.340-346, 10.1109/ICNSC.2013.6548761 . hal-00786270

\section{HAL Id: hal-00786270 https://hal.science/hal-00786270}

Submitted on 8 Feb 2013

HAL is a multi-disciplinary open access archive for the deposit and dissemination of scientific research documents, whether they are published or not. The documents may come from teaching and research institutions in France or abroad, or from public or private research centers.
L'archive ouverte pluridisciplinaire HAL, est destinée au dépôt et à la diffusion de documents scientifiques de niveau recherche, publiés ou non, émanant des établissements d'enseignement et de recherche français ou étrangers, des laboratoires publics ou privés. 


\title{
Motorcycle Maximal Safe Speed in Cornering Situation
}

\author{
H. Slimi and D. Ichalal and H. Arioui and S. Mammar
}

\begin{abstract}
In this paper, we present a new method for the computation of the maximal authorized motorcycle speed in curves. The three main actors which are the vehicle, the driver and the infrastructure are taken into account. The vehicle dynamics are represented by a dynamic four degrees of freedom model which includes the vehicle's longitudinal slip and side slip angle. The driver behavior model considers his ability in deceleration maneuvers according to the mobilized friction. The infrastructure characteristics introduce a precise handling of the road geometry and of the maximal available friction.
\end{abstract}

\section{INTRODUCTION}

The number of death in road accidents has experienced a huge reduction of about $50 \%$ during the last decade. However analysis of accident statistics shows that the number of death when a motorcycle is involved has not decreased and even increased. In fact, the motorcycle remains a particularly dangerous mode of transport: the number of death is still very high, and if one takes account of the number of traveled kilometers, the risk of death for a motorcycle rider is 21 times higher than that of other transportation modes. In additions, motorcycles and scooters become more and more popular for urban an suburban displacement in European countries. Associated traveled kilometers and number of vehicles sold is continuously increasing.

Thus France as well as Europe have launched several research programs in order to study motorcycle dynamics and detect precursor indicators of imminent accident. Notice that good interactions between the rider-the motorcycle and the infrastructure is an important factor. This paper is focused on the development of a computational method of the maximal authorized speed for a motorcycle driving the upcoming curve.

The interaction of the three actors which are the vehicle, the rider and the infrastructure are taken into account. The motorcycle dynamics are represented by a model which includes longitudinal load transfer. The rider behavior considers his ability in deceleration while the infrastructure characteristics introduce a precise handling of the road geometry (curvature, slope and road bank angle) and the maximal available friction. These parameters even if they are not actually available for measurement could be provided through ADASE maps for example.

The remaining of the paper is organized as follow: the problem statement is provided in section (2). Existing speed models in curves are reviewed in section (3) while the proposed speed profile model is detailed in section (4).

Authors are with IBISC Lab, University of Evry Val d'Essonne, 40 rue du Pelvoux, Evry, 91020, France. hichem.ariouidibisc.univ-evry.fr
Simulation results are provided is section (5). The conclusion is given in section (6) wrap up the paper.

\section{OVERVIEW ON LIMIT SAFE MOTORCYCLE PROFILE IN CURVE}

The models on safe profile we discuss in this section are mostly specific to cars (four-wheeled ones). Nevertheless, few models exist for motorcycles. These last were initially concerned the admissible roll angle and speed limits to perform a turn safely [10].

It should be noted that the speed limits that we find on our roads and highways are based on studies conducted for cars than any other category must fulfill (motorcycles) or even reduce it (truck). Also, we can find this type of requirement in terms of infrastructure, sometimes poorly suited for motorcycles, [2].

Among the earliest models, limiting the car's safe speed, is the one proposed by [12] (we call it : simple model). This model is based on a quasi-static study of a car with a mass $M$ moving at $v_{x}$ longitudinal speed taking a turn of $\rho$ curvature. The limit safe speed, neglecting any parameters other than the curvature, is governed by:

$$
v_{x_{\max }}=\sqrt{\frac{g \mu_{\text {lat }}}{\rho}}
$$

Where $\mu_{\text {lat }}$ is the maximum available lateral friction and $g$ is the gravity acceleration.

Unfortunately, this speed model does not contain any other element such as: instant road adhesion, road geometry (slope and elevation), etc. In addition, the curvature radius is assumed constant, what is wrong, at least at the beginning and the exit of turn. However, the centrifugal force depends on the curvature dynamics, which we try to proof afterwards.

In the US, National Highway Traffic Safety Administration (NHTSA) calculates the car's limit speed when cornering based on the following formula [11]:

$$
v_{x_{\max }}=\sqrt{\frac{g}{\rho} \cdot \frac{\phi_{d}+\mu_{\text {lat }}}{1-\phi_{d} \mu_{\text {lat }}}}
$$

Unlike the previous formula takes into account another aspect of the road geometry, ie the bank angle. However, the linearized formula seems to ignore the vehicle angle roll. For motorcycle, the roll angle can reach $30^{\circ}$ in some cases, and therefore absorbs a large part of the lateral acceleration or centrifugal force. Thereafter, we propose a model adapted for bike, taking into account the roll angle and the road slope also. 
As mentioned previously, very few models for setting the speed limit or eligible roll angle, when cornering, have been proposed. Generally, a roll angle providing an outlet to safely turn depends strongly on the road geometry (curvature, slope and bank angles), the road conditions (adhesion, weather), the driver's experience and dynamic states of the vehicle, [10], [12]. Based on the same assumptions (under constant longitudinal speed, $v_{x}$ ) than for cars, [10] proposed a model for limited the admissible roll angle $\phi_{d e s}$ :

$$
\left|\phi_{\text {des }}-\phi_{d}\right|<\arctan \left(\mu_{\text {limit }}\right)
$$

\section{MODELS ENHANCEMENT}

\section{A. Simple Model}

In this section, we present improvements to previous models. For the simple one (1), we illustrate the impact of curvature radius and its "dynamics" on the safe speed limitation. Indeed, the impact of this road parameter (first and second derivative) is particularly important for two-wheelers, because, for example, of the ability of drivers to change the trajectory abruptly.

Consider a material point $P$, hence, the influence of moments is ignored, with mass $M$ rotating around the $z$ axis with angular velocity $\omega$, Figure (1). The trajectory is defined, among others, by its curvature $\rho(t)=\frac{1}{R(t)}$ and its first derivative $\dot{\rho}(t)$ (assumed continuous and differentiable).

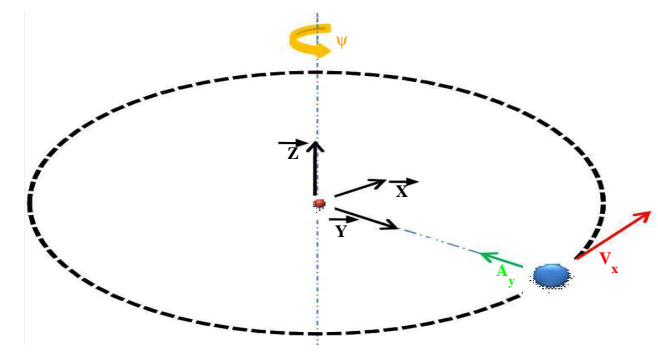

Fig. 1. Kinematic parameters of a point $P$ in circular motion

Under these conditions, we have:

$$
\left\{\begin{array}{l}
\frac{d \vec{y}}{d t}=\dot{\psi} \vec{x} \\
\frac{d \vec{x}}{d t}=-\dot{\psi} \vec{y} \\
\frac{d \vec{z}}{d t}=0
\end{array}\right.
$$

The point $P$ coordinates are given by:

$$
\overrightarrow{O P}=R(t) \vec{y}=\frac{1}{\rho(t)} \vec{y}
$$

We can express the point motion, speed $\vec{v}_{x}=v_{P / O}$ and acceleration $\vec{a}_{y}=\vec{y}=\vec{a}_{P / O}$, by the successive derivatives, as follows:

$$
\left\{\begin{array}{l}
\vec{v}_{P / O}=\frac{d R(t)}{d t} \vec{y}+R(t) \dot{\psi} \vec{x}=\frac{-\dot{\rho}(t)}{\rho(t)^{2}} \vec{y}+\frac{1}{\rho(t)} \dot{\psi} \vec{x} \\
\vec{a}_{P / O}=\left(\frac{d^{2} R(t)}{d t^{2}}-R(t) \dot{\psi}^{2}\right) \vec{y}+\left(2 \frac{d R(t)}{d t} \dot{\psi}+R(t) \ddot{\psi}\right) \vec{x}
\end{array}\right.
$$

This equation can be rewritten as a function of $v_{x}$ speed by substituting $\psi$ by $\frac{1}{R(t)} v_{x}$. The equation (6) has two important terms, namely:

- The first term obtained by projection on $\vec{y}$ axis represents the centrifugal acceleration (force): $\left(\frac{d^{2} R(t)}{d t^{2}}-\frac{v^{2}}{R(t)}\right)$. It is noted that in the case of a circular road type (constant curvature $\dot{\rho}=0$ ) or a clothoid, $\dot{\rho}=$ constant, acceleration (or force) depends only on the centrifugal speed and the instantaneous curvature value. Moreover, the curvature dynamics can affect the safe speed limit, especially at the beginning and the end of turn, where the radius and its first derivative are variable.

- The second term obtained by projection on $\vec{x}$ axis represents the tangential acceleration: $\left(2 \frac{d R(t)}{d t} \dot{\psi}+R(t) \dot{\psi}\right)$.

The highest safe speed in a curve, can be rewritten as follows:

$\ddot{y}=-\frac{d^{2} R(t)}{d t^{2}}+\frac{V_{x}^{2}}{R(t)}=g \mu_{\text {lat }} \Rightarrow v_{x_{\max }}=\sqrt{\frac{\ddot{R}(t)+g \mu_{\text {lat }}}{\rho(t)}}$

with:

$$
\frac{d^{2} R(t)}{d t^{2}}=\frac{2 \rho \dot{\rho}^{2}-\rho^{2} \ddot{\rho}}{\rho^{4}}
$$

Also, the impact of curvature dynamics may be more important in the determination of the under or over-steering cases.

\section{B. NHTSA Model}

As stated before, equation (2) takes into account the slope angle, road curvature and adherence and seems to ignore the roll angle of the vehicle itself.

If we consider a single track, the equilibrium will be governed by the following equations:

$$
\left\{\begin{array}{l}
F_{x}=R \sin \left(\phi_{p}\right) \cos \left(\alpha+\phi+\phi_{d}\right) \\
R \cos \left(\phi_{p}\right) \sin \left(\alpha+\phi+\phi_{d}\right)=M \rho v_{x}^{2} \\
R \cos \left(\phi_{p}\right) \cos \left(\alpha+\phi+\phi_{d}\right)=M g
\end{array}\right.
$$

where $: \tan (\alpha)=\mu_{\text {lat }}$.

Then, we can express, after light calculations, the maximum safe speed by:

$$
v_{x_{\text {max }}}=\sqrt{\frac{g}{\rho} \frac{\tan \left(\phi_{d}\right)+\frac{\tan (\phi)+\mu_{\text {lat }}}{1-\tan (\phi) \mu_{\text {lat }}}}{1-\tan \left(\phi_{d}\right) \frac{\tan (\phi)+\mu_{\text {lat }}}{1-\tan (\phi) \mu_{\text {lat }}}}}
$$

We can notice that the slope angle has no effect on the speed limit calculation $v_{x_{\max }}$. Also, the bank and the roll angles have the same effect on $v_{x_{\max }}$ synthesis. This relationship gives highest safe speed limit for an admissible roll angle which could be calculated by various methods [1], [11]. 


\section{New Approach For Limit Speed Calculation}

The proposed approach takes into account the driver behavior and the road geometry. Also, the main idea of the limit speed calculation is based on motorcycle dynamics:

- A two bodies motorcycle dynamic model which takes into account the forces of tire/road contact and the load transfer due to the longitudinal accelerations.

- Lateral and longitudinal mobilized frictions on each wheel are computed. The highest safe speed is defined considering the maximum friction. As we consider a four degrees (roll, steer, yaw and longitudinal dynamics) of freedom model, its speed is computed by resolving the maximum of the two tires mobilized frictions.

- The driver behavior (acceleration/deceleration profile).

\section{A. Motorcycle Dynamic Model}

This model has two joined rigid bodies and is inspired from those presented in [4], [5], [6], [7], [8].

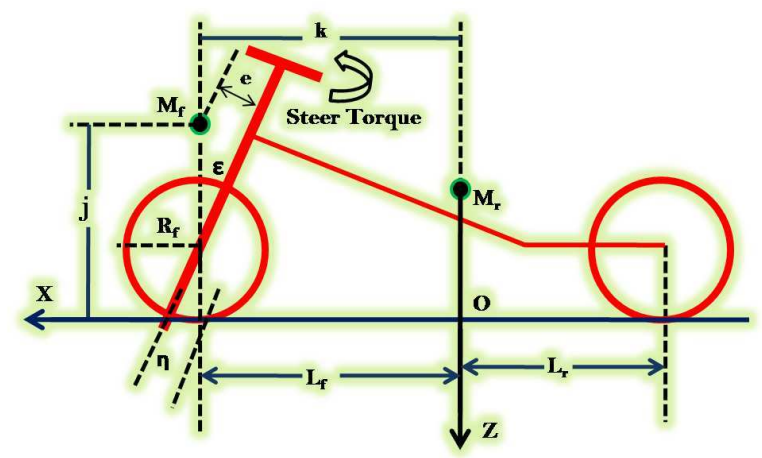

Fig. 2. Schematic diagram of the Motorcycle

The longitudinal dynamics is given by:

$$
\begin{aligned}
& M(\ddot{x}-\dot{y} \dot{\psi})-\left(M_{f} j+M_{r} h\right) \dot{\phi} \dot{\psi}^{2}-\left(M_{f} \text { e } \cos \varepsilon\right) \dot{\delta}^{2} \\
& -F_{\text {aerolong }}-M g \sin \phi_{d} \sin \phi_{p}=\sum F_{x i}
\end{aligned}
$$

Where, $\sum F_{x i}$ represents the longitudinal forces acting on the tires according to the longitudinal slip. $F_{\text {aerolong }}$ is the aerodynamic force in the longitudinal direction.

$$
\sum F_{x i}=F_{x f}+F_{x r}=\sum K_{p i} \lambda_{i}
$$

Where $i=1,2 . K_{p i}$ is the longitudinal stiffness coefficient of tires, $\lambda_{i}$ is the tires longitudinal slip.

The lateral dynamics is described by [1]:

$$
\begin{aligned}
& M(\ddot{y}+\dot{x} \dot{\psi})+\left(M_{f} j+M_{r} h\right) \ddot{\phi}+M_{f} k \ddot{\psi}+M_{f} e \ddot{\delta} \\
& -F_{\text {aerolat }}+M g \cos \phi_{p} \sin \phi_{d} \\
& =\sum F_{y i} \\
& \left(M_{f} j+M_{r} h\right) \ddot{y}+a_{1} \ddot{\phi}+a_{2} \ddot{\psi}+a_{3} \ddot{\delta}+a_{4} \dot{x} \dot{\psi}+a_{5} \dot{x} \dot{\delta} \\
& =\sum M_{x} \\
& M_{f} k(\ddot{y}+\dot{x} \dot{\psi})+a_{2} \ddot{\phi}+b_{1} \ddot{\psi}+b_{2} \ddot{\delta}-b_{3} \dot{x} \dot{\phi}-a_{5} \dot{x} \dot{\delta} \\
& =\sum M_{z} \\
& M_{f} e \ddot{y}+a_{3} \ddot{\phi}+c_{1} \ddot{\psi}+c_{2} \ddot{\delta}-a_{5} \dot{x} \dot{\phi}+c_{3} \dot{x} \dot{\psi} \\
& =\sum M_{s}
\end{aligned}
$$

Where $a_{i}, b_{j}$ and $c_{k}$ are defined in the annex section (VIII). We have:

$$
\begin{aligned}
& \sum M_{x}=\left(M_{f} j+M_{r} h\right) g \sin \phi-\left(\eta F_{z f}-M_{f} e g\right) \sin \delta \\
& \sum M_{z}=l_{f} F_{f}-l_{r} F_{r}-\tau \cos \varepsilon \\
& \sum M_{s}=-\left(\eta F_{z f}-M_{f} \text { e } g\right) \sin \phi-\left(\eta F_{z f}-M_{f} \text { e } g\right) \sin \varepsilon \sin \delta+\tau
\end{aligned}
$$

Where, $\sum F_{y i}$ are the lateral forces exerted on each tire. We can express the lateral forces on each tire as, [1]:

$$
\left\{\begin{array}{l}
F_{y f}=-C_{f 1} \alpha_{f}+C_{f 2} \gamma_{f} \\
F_{y r}=-C_{r 1} \alpha_{r}+C_{r 2} \gamma_{r}
\end{array}\right.
$$

Where, $C_{f i}$ and $C_{r j}$ are the lateral stiffness and the camber coefficients of the tire.

On the other hand, the slip angles of the front and rear tires are given by:

$$
\left\{\begin{aligned}
\alpha_{f} & =\left(\frac{v_{y}+L_{f} \dot{\psi}-\eta \dot{\delta}}{v_{x}}\right)-\delta \cos \varepsilon \\
\alpha_{r} & =\left(\frac{v_{y}-L_{r} \dot{\psi}}{v_{x}}\right)
\end{aligned}\right.
$$

$L_{f}$ (resp. $L_{r}$ ) represents the distance between the center of mass and the front axis (resp. rear axis).

The camber angles of front and rear tires are given by [1]:

$$
\left\{\begin{array}{l}
\gamma_{f}=\phi+\delta \sin \varepsilon \\
\gamma_{r}=\phi
\end{array}\right.
$$

Finally, we can express the lateral forces on the two wheels as follows:

$$
\left\{\begin{array}{l}
Y_{f}=C_{f}\left(\delta \cos \varepsilon-\frac{v_{y}+L_{f} \dot{\psi}-\eta \dot{\delta}}{v_{x}}\right)+C_{f 2}(\phi+\delta \sin \varepsilon) \\
Y_{r}=C_{r 1}\left(\frac{L_{r} \dot{\psi}-v_{y}}{v_{x}}\right)+C_{r 2} \phi
\end{array}\right.
$$

Under dynamical conditions, load can transfer on the front wheel when braking or on the rear wheel during accelerations/decelerations phases. In this cases, normal forces on the two tires can be obtained as follows:

$$
\left\{\begin{array}{l}
F_{z f}=\frac{M_{f}+M_{r}}{L_{f}+L_{r}}\left(L_{r} g \cos \phi_{p} \cos \phi_{d}-h \ddot{x}\right) \\
F_{z r}=\frac{M_{f}+M_{r}}{L_{f}+L_{r}}\left(L_{f} g \cos \phi_{p} \cos \phi_{d}+h \ddot{x}\right)
\end{array}\right.
$$

Where, $h$ is the height of the center of mass, $L_{f}$ (resp. $L_{r}$ ) corresponding to the dimensions of vehicle configuration (horizontal dimensions of the overall center of gravity relative to the center of the front wheel, rear respectively). $F_{z f}$ (resp. $F_{z f}$ ) represent normal forces applied on the front tire (resp. rear tire).

\section{B. Tire Road Friction}

The tire dynamic behavior is very complex and is linear only under certain restricted conditions of drive. We can observe various phenomena like skidding and blocking.

The Coulomb friction model is used to obtain the mobilized friction $\mu_{m o b}$. Thus the transversal force $F_{t}$ and the normal force $F_{n}=F_{z f}+F_{z r}$ are such that: 


$$
\mu_{m o b}=\frac{F_{t}}{F_{n}} \leq \mu_{\max }
$$

Where $\mu_{\max }$ is the maximum available friction.

Since:

$$
F_{t}^{2}=F_{\text {lat }}^{2}+F_{\text {long }}^{2}
$$

We have:

$$
\begin{cases}\mu_{m o b}^{2} & =\frac{F_{\text {lat }}^{2}+F_{\text {long }}^{2}}{F_{n}^{2}}=\mu_{\text {lat }}^{2}+\mu_{\text {long }}^{2} \\ \mu_{\text {lat }} & =\frac{F_{\text {lat }}}{F_{n}} \\ \mu_{\text {long }} & =\frac{F_{\text {long }}}{F_{n}}\end{cases}
$$

To calculate $\mu_{\text {lat }}$ and $\mu_{\text {long }}$, we use the simplified model of the motorcycle in absolute frame based on previous model, including lateral and longitudinal equations, as follows:

$$
\left\{\begin{array}{l}
M \ddot{y}=\left(Y_{f}+Y_{r}\right)-M g \sin \left(\phi+\phi_{d}\right) \\
F_{x}=M \ddot{x}-M g \sin \phi_{p} \\
I_{z} \ddot{\psi}=\left(L_{f} Y_{f}-L_{r} Y_{r}\right) \cos \left(\phi+\phi_{d}\right)
\end{array}\right.
$$

Then, front and rear lateral forces expressed by:

$$
\left\{\begin{array}{l}
Y_{f}=\frac{L_{r} M \ddot{y} \cos \left(\phi+\phi_{d}\right)+L_{r} M g \sin \left(\phi+\phi_{d}\right) \cos \left(\phi+\phi_{d}\right)+I_{z} \ddot{\psi}}{\left(L_{f}+L_{r}\right) \cos \left(\phi+\phi_{d}\right)} \\
Y_{r}=\frac{L_{f} M \ddot{y} \cos \left(\phi+\phi_{d}\right)+L_{f} M g \sin \left(\phi+\phi_{d}\right) \cos \left(\phi+\phi_{d}\right)-I_{z} \ddot{\psi}}{\left(L_{f}+L_{r}\right) \cos \left(\phi+\phi_{d}\right)}
\end{array}\right.
$$

After light calculations and using the vertical forces (19), lateral and longitudinal frictions on each wheel are given by:

$$
\left\{\begin{array}{l}
\mu_{\text {lat } f}=Y_{f} / F_{z f} \\
=\frac{L_{r} M \ddot{y} \cos \left(\phi+\phi_{d}\right)+L_{r} M g \sin \left(\phi+\phi_{d}\right) \cos \left(\phi+\phi_{r}\right)+I_{z} \ddot{\psi}}{\left(L_{r} M g \cos \left(\phi_{p}\right)-h F_{x}\right) \cos \left(\phi+\phi_{d}\right)} \\
\mu_{\text {latr }}=Y_{r} / F_{z r} \\
=\frac{L_{f} M \ddot{y} \cos \left(\phi+\phi_{d}\right)+L_{f} M g \sin \left(\phi+\phi_{d}\right) \cos \left(\phi+\phi_{r}\right)-I_{z} \ddot{\psi}}{\left(L_{f} M g \cos \left(\phi_{p}\right)+h F_{x}\right) \cos \left(\phi+\phi_{d}\right)}
\end{array}\right.
$$

The previous system can be simplified by:

$$
\left\{\begin{array}{c}
\mu_{\text {lat } f}=\frac{\mu_{\text {lat }} \cos \left(\phi+\phi_{d}\right)+\frac{I_{z} \ddot{\psi}}{L_{r} M g}}{\left(\cos \phi_{p}-\frac{h F_{x}}{L_{r} M g}\right) \cos \left(\phi+\phi_{d}\right)} \\
\mu_{\text {latr }}=\frac{\mu_{\text {lat }} \cos \left(\phi+\phi_{d}\right)-\frac{I_{z} \ddot{\psi}}{L_{f} M g}}{\left(\cos \phi_{p}+\frac{h F_{x}}{L_{f} M g}\right) \cos \left(\phi+\phi_{d}\right)}
\end{array}\right.
$$

We assume here that $\frac{I_{z} \ddot{\psi}}{L_{r} M g \cos \left(\phi+\phi_{d}\right)}$ is very small against $\mu_{\text {lat }}$. Then, we can rewrite the previous system as :

$$
\left\{\begin{array}{l}
\mu_{\text {lat } f}=\frac{\mu_{\text {lat }}}{\left(1-\frac{h a_{x}}{L_{r} g}\right) \tan \left(\phi_{p}\right)} \\
\mu_{\text {latr }}=\frac{h \mu_{\text {lat }}}{\left(1+\frac{h a_{x}}{L_{f} g}\right) \tan \left(\phi_{p}\right)} \\
\mu_{\text {lat }}=\frac{a_{y}}{g}+\sin \left(\phi+\phi_{d}\right) \\
\mu_{\text {long }}=\frac{a_{x}}{g}-\sin \left(\phi_{p}\right)
\end{array}\right.
$$

The highest safe speed is defined considering the maximum mobilized friction. As we consider a two-wheeled vehicle model, the aim is to find the maximum between the two mobilized frictions, so:

$$
\mu_{\text {max }}=\max \left(\sqrt{\mu_{\text {latf }}^{2}+\mu_{\text {longf }}^{2}}, \sqrt{\mu_{\text {latr }}^{2}+\mu_{\text {longr }}^{2}}\right)
$$

\section{Driver Behavior}

The driver is unceasingly obliged, according to the information taken from the environment, to define an appropriate speed and position to the encountered situation [3]. Here, the capacities of the driver in acceleration are taken into account. The driver cannot mobilize the same level of acceleration into longitudinal and lateral directions. For this reason, we have to distinguish between the maximum friction in longitudinal and lateral modes:

$$
\begin{array}{r}
1=\max \left(\sqrt{\left(\frac{\mu_{\text {lat } f}}{\mu_{\text {latmax }}}\right)^{2}+\left(\frac{\mu_{\text {longf }}}{\mu_{\text {longmax }}}\right)^{2}},\right. \\
\left.\sqrt{\left(\frac{\mu_{\text {latr }}}{\mu_{\text {latmax }}}\right)^{2}+\left(\frac{\mu_{\text {longr }}}{\mu_{\text {longmax }}}\right)^{2}}\right)
\end{array}
$$

For comfort and safety reasons, and under good weather conditions, a driver generally does not mobilize all the available lateral and/or longitudinal friction. Therefore we represent the driver behavior with through two dimensionless coefficients $\lambda_{\text {lat }}, \lambda_{\text {long }}$ and a maximum friction $\mu_{\text {max }}$ [3].

$$
\mu_{\text {latmax }}=\lambda_{\text {lat }} \cdot \mu_{\max } \quad \text { and } \quad \mu_{\text {longmax }}=\lambda_{\text {long }} \cdot \mu_{\max }
$$

We consider that the driver behavior varies proportional to $\frac{1}{\mu_{\max }}$.

$$
\left\{\begin{array}{l}
\lambda_{\text {long }}=\Delta \lambda_{\text {long }} \mu_{\text {max }}-\lambda_{\text {slip-long }} \\
\lambda_{\text {lat }}=\Delta \lambda_{\text {lat }} \mu_{\text {max }}-\lambda_{\text {slip-lat }}
\end{array}\right.
$$

With:

$$
\left\{\begin{array}{l}
\Delta \lambda_{\text {long }}=\lambda_{\text {dry-long }}-\lambda_{\text {slip-long }} \\
\Delta \lambda_{\text {lat }}=\lambda_{\text {dry-lat }}-\lambda_{\text {slip-lat }}
\end{array}\right.
$$

Where, $\lambda_{d r y}$ (resp. $\lambda_{\text {slip }}$ ) represents the maximum friction that the driver can mobilize on a dry road (resp. slipping one)

\section{Generation of Speed Profile}

Considering the equilibrium state, we can make the following approximations:

$$
\left\{\begin{array}{l}
\phi=\tan ^{-1}\left(\frac{\rho v_{x}^{2}}{g}\right) \\
a_{x}=\ddot{x}=\frac{d v_{x}}{d t}=v_{x} \frac{d v_{x}}{d s} \\
\dot{\psi}=\rho v_{x}
\end{array}\right.
$$

Where, $s$ is the road curvilinear coordinate. Finally $\mu_{\text {latf }}$ and $\mu_{\text {latr }}$ may be given in a more compact form, in braking and accelerating cases, as:

In a simpler form:

$$
\left\{\begin{array}{l}
1=\left(\frac{\frac{\ddot{y}}{g}+\sin \left(\phi+\phi_{d}\right)}{\eta_{1}}\right)^{2}+\eta_{2}^{2} \\
1=\left(\frac{\frac{\ddot{y}}{g}+\sin \left(\phi+\phi_{d}\right)}{\eta_{3}}\right)^{2}+\eta_{2}^{2}
\end{array}\right.
$$

With:

$$
\left\{\begin{array}{l}
\eta_{1}=\left(\lambda_{\text {lat }} \mu_{\max }\right)\left(1+\frac{h}{L_{f}}\left(\frac{v_{x}}{g} \frac{d v_{x}}{d s}-\tan \phi_{p}\right)\right) \\
\eta_{2}=\left(\frac{1}{\lambda_{\text {lat }} \mu_{\max }}\right)\left(\frac{v_{x}}{g} \frac{d v_{x}}{d s}-\tan \phi_{p}\right) \\
\eta_{3}=\left(\lambda_{\text {lat }} \mu_{\max }\right)\left(1-\frac{h}{L_{r}}\left(\frac{v_{x}}{g} \frac{d v_{x}}{d s}-\tan \phi_{p}\right)\right)
\end{array}\right.
$$


By developing the previous equations (34), the maximum speed $v_{x i}$ at each wheels is given by solving the following equation system of equations as shown by the following block diagram (Figure 3).

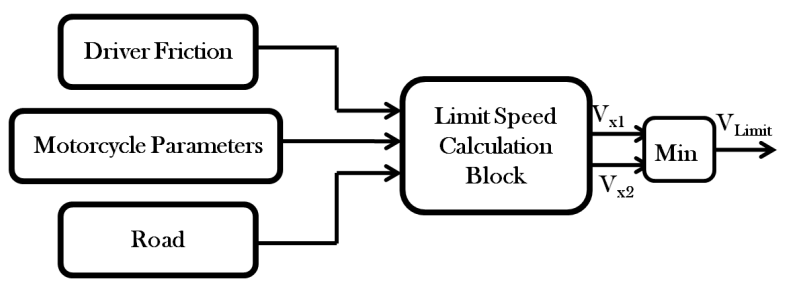

Fig. 3. Block diagram for limit speed calculation

Then, the highest safe speed $v_{\text {limit }}$, in curve, is obtained by solving: $v_{\text {limit }}=\min \left(v_{x 1}, v_{x 2}\right)$.

This model speed is function of intrinsic motorcycle parameters, roadway parameters and driver behavior. Also, it takes into account the vehicle side slip, and longitudinal dynamics by considering the longitudinal slip on each wheel.

\section{ROAD PARAMETERS ESTIMATION AND DYNAMIC STATES RECONSTRUCTION}

To deploy such safety systems or function on motorcycles, one has to know reliably what the current state of the vehicle and road parameters are. Various sensors can be used (e.g. lateral acceleration, yaw rate, wheel speeds), which are already used for four-wheeled vehicle applications. However, the major difference concerns the roll angle which can exceed $40^{\circ}$ in some limit cases or lateral speed (due to initial condition issue).

Roll angle is the main characteristic of the Motorcycle lateral dynamics. A good control of motorcycle motions requires an accurate assessment of this quantity and for safety applications also the risk of sliding or friction loss need to be considered. Direct measurement of roll angle and tire slip is not available or very expensive as for the lateral speed (corevit sensor). Some previous work [14] and [13] have addressed these challenges based on estimation and/or observation techniques of these dynamic parameters.

Regarding to road parameters estimation, two approaches can be implemented. The fist one uses observation techniques based on unknown input estimation. Indeed, as shown previously, the function calculating the safe authorized maximal speed depends on some motorcycle parameters. Due to economic and/or technique reasons these parameters are not accessible to measure. Observers can then provide an estimation of them. For that purpose, in future works, we consider a nonlinear model of the motorcycle in order to provide an accurate and robust estimation of the parameters which allows reducing false alarms and to give a good estimation of the safe authorized maximal speed in curves. The second approach is based on available data given by cartographies.

This issues and their real time implementation will be addressed in future works.

\section{Simulation Results}

In this section, we test the new safe speed model we have proposed and the impact of inherent parameters. Also, we compare it with existing models (1 and 2).

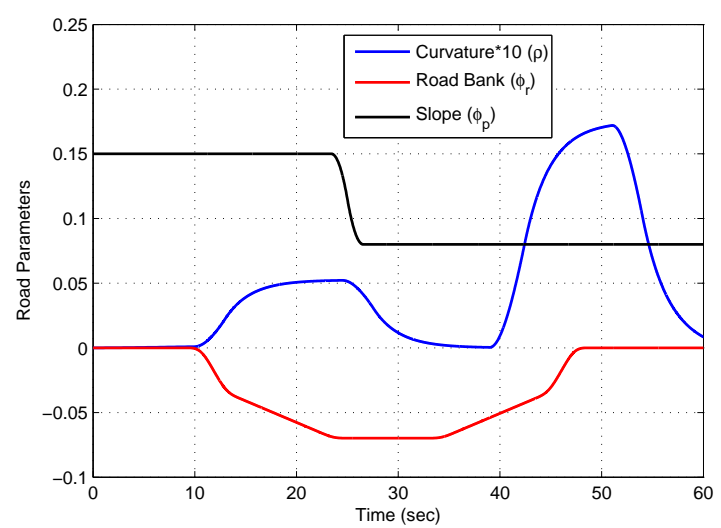

Fig. 4. Road Parameters

simulation tests were conducted under the same conditions: the motorcycle moves on a road with two curves in the same direction with positive curvature radius of different sizes (blue line on Figure 4). The variations of slope and bank are chosen to highlight the impact of each on the limit of the safe speed (Figure 4). We also limited the longitudinal velocity to about $40 \mathrm{~m} / \mathrm{s}$ in straight line. For all simulation conditions, adhesions coefficients $\mu_{\max }$ are fixed around 0.7 .

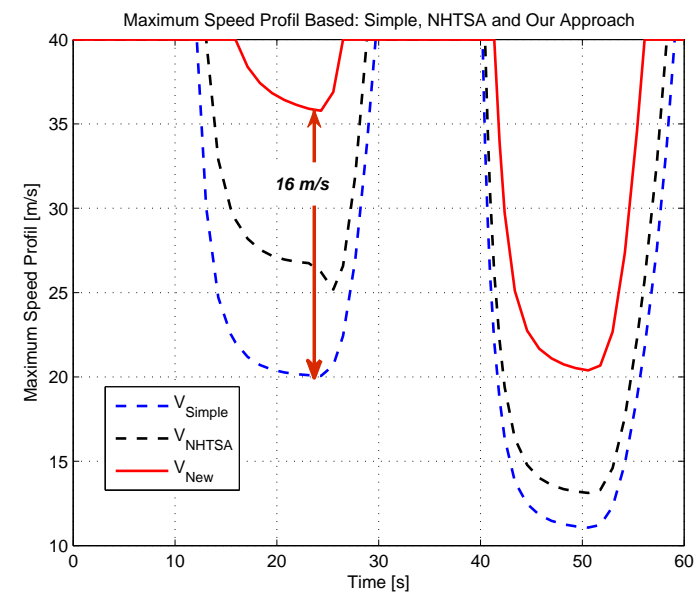

Fig. 5. Maximum Speed Profile synthesized by Simple (1), NHTSA (2) and the proposed (34) approaches

Figure (5) illustrates the various speed limits obtained by the three models (equations: 1, 2 and 34). The difference between the speed synthesized by our approach and those given by the other two approaches is significant. Indeed, this speed difference (between the first and third model) can reach, in some cases, $16 \mathrm{~m} / \mathrm{s}$. The first observation can be done on the impact of the bank angle on the NHTSA and the proposed models in this paper. The second remark is made on 
the discrepancy in these two models, where the motorcycle dynamics (load transfer, geometry and inertia of the bike, etc.) and the driver behavior parameters are very important.

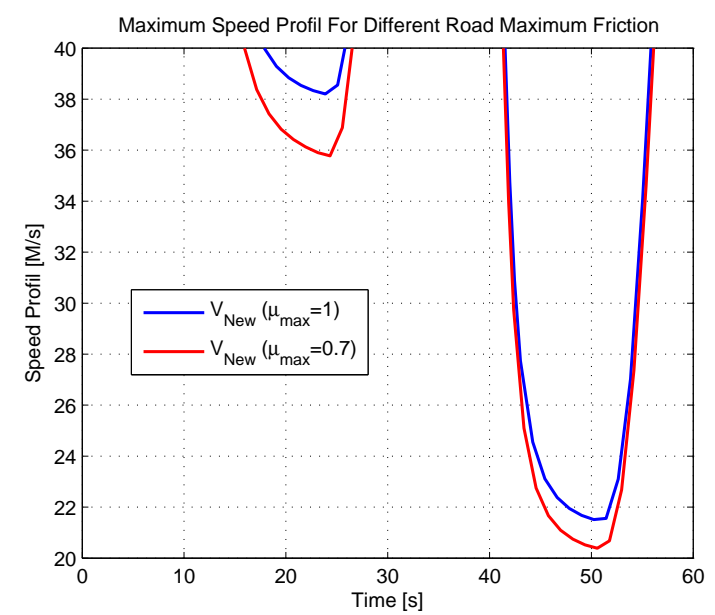

Fig. 6. Maximum Speed Profile For Different Road Maximum Friction

Figure (6) shows the influence of the maximum road adhesion and confirms that high adhesion results in an increased high safe speed. This speed is maximum when $\lambda_{i}=1$.

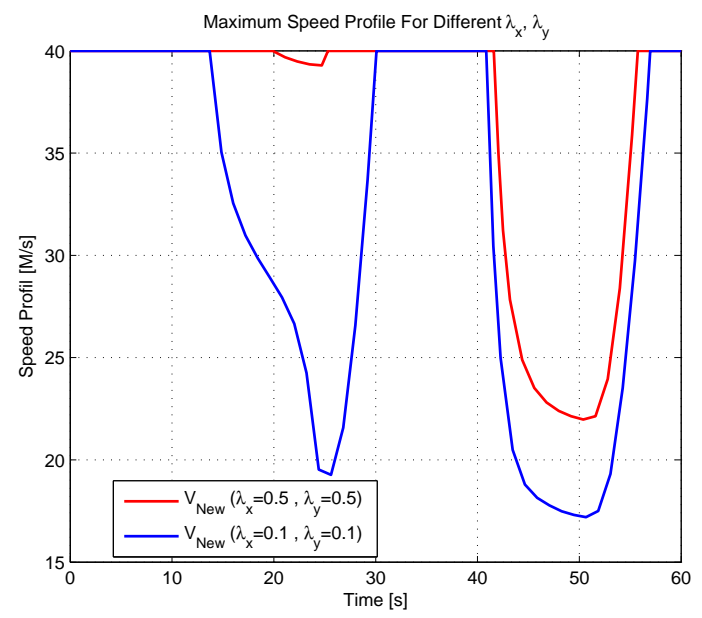

Fig. 7. Maximum Speed Profile For Different $\lambda_{\text {longx }}, \lambda_{\text {lat }}$

Figure (7) shows, the influence of the driver behaviour $\left(\lambda_{\text {long }}\right.$, and $\left.\lambda_{\text {lat }}\right)$ on limit safe speed. When $\lambda_{\text {long }}$ and $\lambda_{\text {lat }}$ ( $\lambda_{\text {long }}=0.1$, and $\left.\lambda_{\text {lat }}=0.1\right)$ decrease the speed drops significantly.

\section{CONCLUSIONS AND FUTURE WORKS}

In this paper, we studied and adapted some existing models for the synthesis of the profile of the highest safe speed when cornering. Moreover, we proposed a new model taking into account the motorcycle dynamics, the road's geometry and driver behavior.
We have shown that existing models (single or NHTSA one) give a safe speed profiles not adapted or suitable for two-wheeled vehicles. These models take into account little important information, for example adherence and geometry of the road (curvature and/or the bank angle). This means that whatever the vehicle be light or heavy (not taking into account the mass and inertia), would not change the safe speed limit. This finding is of course false, since the centrifugal force is proportional to the vehicle mass. On the other hand, we found that a safe speed profile giving by the simple model can depend on curvature dynamics (second derivative) and thus impacting, according to the road shape, the safe speed profile. This issue will be studied soon.

In addition, we proposed a new "dynamic" model to calculate the motorcycle speed limit when cornering. This model does not take into account the quasi-static conditions as the two previous models. The motorcycle dynamics (load transfer, roll and camber angles), road infrastructure (adhesion, curvature, bank angle) and driver behavior are supported in the proposed model. The effect of these parameters is significant, since we have a safe speed difference, under the same conditions of simulation, about $16 \mathrm{~m} / \mathrm{s}$ with the simple model and about $9 \mathrm{~m} / \mathrm{s}$ with NHTSA model.

In future work, we plan to synthesize a limit roll profile in cornering situations. It should be noted that the roll dynamics is one of the most important degrees of freedom. In addition, dynamic states and road profile estimation will be addressed thus the impact of road traffic.

\section{REFERENCES}

[1] H. Slimi, H. Arioui, L. Nouveliere and S. Mammar, "Advanced Motorcycle-Infrastructure-Driver Roll Angle Profile for Loss Control Prevention", 12th International IEEE Conference on Intelligent Transportation Systems, St. Louis, Missouri, USA, October 3-7, 2009

[2] B. Amans, M. Moutreuil, "RIDER : Recherche sur les accidents impliquant un deux roues motoris", Reaserch Report of RIDER Project, Mars 2005.

[3] Glaser, S., 2004, “ Modelisation et analyse d'un vehicule en trajectoire limites Application au developpement de systemes d'aide a la conduite". PhD thesis, University of Evry Val d'Essonne (France).

[4] C. Koenen, "The dynamic behaviour of motorcycles when running straight ahead and when cornering," Ph.D. dissertation, Delft University of Technology, 1983.

[5] D. J. N. Limebeer and R. S. Sharp, "Bicycles, motorcycles and models," IEEE Control Systems magazine, vol. 26, no. 5, pp. 34-61, 2006.

[6] R. S. Sharp and D. J. N. Limebeer, "A motorcycle model for stability and control analysis", Multibody System Dynamics, vol. 6, no. 2, pp. 123-142, 2001.

[7] V. Cossalter and R. Lot, "A motorcycle multi-body model for real time simulations based on the natural coordinates approach", Vehicle System Dynamics, vol. 37, no. 6, pp. 423-447, 2002.

[8] H. Slimi, H. Arioui, L. Nouveliere and S. Mammar, "Preventive Safety: Warning System for Control Loss of Two-Wheeled Vehicles", Informatics, Integrative Biology and Complex Systems, Zarzis, Tunisia on 23-25 March 2009.

[9] S Hima, L Nehaoua, N Seguy and H Arioui, "Motorcycle Dynamic Model Synthesis for Two Wheeled Driving Simulator", IEEE Intelligent Transportation Systems Conference, pp. 812-817, 2007.

[10] S. Evangelou, David J. N. Limebeer. and Maria Tomas Rodriguez. "Infuence of Road Camber on Motorcycle Stability". Electrical and Electronic and Mechanical Engineering Imperial College London, London SW7 2AZ, U.K. 
[11] D. Pomerleau, T. Jochem, C. Thorpe, P. Batavia, D. Pape, J. Hadden, N. McMillan, N. Brown, and J. Everson. "Run-off-road collision avoidance using ivhs countermeasures", Technical Report DOT HS 809 170, U.S. Department of Transportation, National Highway Traffic Safety Administration, 1999.

[12] D. Karnopp and R. Hibbard. "Optimum Roll Angle behavior for Tilting Ground Vehicle", ASME Journal of Transportation Systems (DSC), vol. 44, pp. 29-37, Novembre 1992.

[13] Stefano, C. and Boniolo, I. and Savaresi, S. "Attitude Estimation of a Motorcycle Via Unscented Kalman Filter", 5th IFAC Symposium on Mechatronic Systems, 13-15 September, 2010. Cambridge, Massachusetts, USA.

[14] Teerhuis, A.P. and Jansen, S.T.H. "Motorcycle State Estimation for Lateral Dynamics", Bicycle and Motorcycle Dynamics, 2022 October 2010, Delft, The Netherlands..

\section{APPENDIX}

$\begin{array}{ll}\phi, \psi, \delta & \text { APPENDIX } \\ F_{x f}, F_{y f} & \text { Lateral and longitudinal front forces } \\ F_{x r}, F_{y r} & \text { Lateral and longitudinal rear forces } \\ F_{z f}, F_{z r} & \text { Front and rear vertical forces } \\ x, y & \text { Longitudinal and lateral displacement } \\ M, M_{f}, M_{r} & \text { Masses of whole, front and rear bodies } \\ \phi_{p}, \phi_{d} & \text { Slope and road bank angles } \\ \rho, R & \text { Road curvature and radius. } \\ I_{x}, I_{z} & \text { Roll and yaw moment of inertia } \\ I_{s}, I_{x z} & \text { Steer and product moment of inertia } \\ \alpha, \gamma & \text { Side slip and camber angles } \\ \tau & \text { Rider applied steering torque } \\ \kappa & \text { Steering damper coefficient } \\ \eta & \text { Trail } \\ \varepsilon & \text { Caster angle } \\ g & \text { Acceleration due to gravity } \\ a_{i}, b_{j}, c_{k} & \text { Motorcycle model parameters based on masses and inertia }\end{array}$

